\title{
LUMINOSITIES OF BARRED AND UNBARRED S0 GALAXIES
}

\author{
Sidney van den Bergh \\ Dominion Astrophysical Observatory, Herzberg Institute of Astrophysics, National \\ Research Council of Canada, 5071 West Saanich Road, Victoria, BC, V9E 2E7, Canada \\ sidney.vandenbergh@nrc.gc.ca
}

Received __; accepted _ 


\begin{abstract}
Lenticular galaxies with $M_{B}<-21.5$ are almost exclusively unbarred, whereas both barred and unbarred objects occur at fainter luminosity levels. This effect is observed both for objects classified in blue light, and for those that were classified in the infrared. This result suggests that the most luminous (massive) S0 galaxies find it difficult to form bars. As a result the mean luminosity of unbarred lenticular galaxies in both $\mathrm{B}$ and IR light is observed to be $\sim 0.4$ mag brighter than than that of barred lenticulars. A small contribution to the observed luminosity difference that is found between SA0 and SB0 galaxies may also be due to the fact that there is an asymmetry between the effects of small classification errors on SA0 and SB0 galaxies. An E galaxy might be misclassified as an S0, or an S0 as an E. However, an E will never be misclassified an SB0, nor will an SB0 ever be called an E. This asymmetry is important because elliptical (E) galaxies are typically twice as luminous as lenticular (S0) galaxies. The present results suggest that the evolution of luminous lenticular galaxies may be closely linked to that of elliptical galaxies, whereas fainter lenticulars might be more closely associated with ram-pressure stripped spiral galaxies. Finally it is pointed out that fine details of the galaxy formation process might account for some of the differences between the classifications of the same galaxy by individual competent morphologists.
\end{abstract}

Subject headings: galaxies: elliptical and lenticular 


\section{INTRODUCTION}

The class of lenticular (S0) galaxies was introduced by Hubble (1936) as a more-or-less speculative means of bridging the morphological chasm between elliptical (E) and spiral (S) galaxies. The definition of the S0 class was later improved and expanded by de Vaucouleurs (1959) and by Sandage (1961). Since then numerous individual lenticular galaxies have been classified on large-scale photographic plates by Sandage \& Tammann (1981), Sandage \& Bedke (1994), de Vaucouleurs et al. (1991) and by Buta, Corwin \& Odewahn (2007). Inter-comparison of the individual classifications of lenticular galaxies by these expert morphologists reveals so much dispersion that King (1992) and Djorgovski (1992) proposed giving up entirely on optical morphological classification and replacing it by a system of measured physical parameters. A less drastic, and perhaps more productive, procedure has been proposed by Laurikainen et al. (2011, 2012), who obtained large diameter images at a wavelength of 2.2 microns which trace the old stellar populations of early-type galaxies in a fashion that is essentially free of the influence of internal extinction and the effects of recent star formation. It is the purpose of the present paper to use these infrared morphological classifications of S0 galaxies by Laurikainen et al., in conjunction with their distances and luminosities assigned by Sandage \& Tammann (1981) [who assumed $H_{o}=50 \mathrm{~km} \mathrm{sec}-1$ $\mathrm{Mpc}^{-1}$ ] to study the properties of the bright relatively nearby lenticular galaxies. For the

sake of convenience the $M_{B_{T}}^{o, i}$ magnitudes of Sandage \& Tammann (1981) will subsequently be referred to as $M_{T}$ The luminosity differences between various classes of galaxies that are discussed in the present paper are, of course, independent of the value of the Hubble parameter adopted by Sandage\& Tammann. 


\section{LUMINOSITIES OF UNBARRED AND BARRED SO GALAXIES}

Laurikainen et al. (2011) have recently published a near-IR atlas of early-type galaxies. Their data are discussed in more detail by Laurikainen et al. (2012). Table 3 of the former paper lists galaxy classifications made on the basis of inspection of 2.2 micron galaxy images. The luminosity distributions of non-barred and barred early-type galaxies in their data are listed in Table 1. Inspection of this table shows that non-barred (SA0) galaxies are typically more luminous than barred (SB0) galaxies. Galaxies which these authors assign to the intermediate class SAB0 are found to be of intermediate luminosity. A Kolmogorov-Smirnov test shows that there is only a $2 \%$ probability that the luminosity distributions of SA0 and of SB0 galaxies in this table were drawn from the same parent population. Table 2 shows that the difference between the frequency distribution of barred and unbarred lenticular galaxies is almost entirely due to the absence of SB0 galaxies that are more luminous than $M_{B}=-21.5$. Table 3 shows a similar effect for the galaxies classified in blue light by Sandage \& Tammann. [It is noted in passing that both of the two most luminous SB0 galaxies in the Revised Shapley-Ames Catalog have $M_{B}=-21.51$.] Inter comparison of Tables 2 and 3 shows that the almost complete absence of luminous barred lenticular galaxies is observed in both blue and infrared light. It is also noted that the difference between the median magnitudes of barred and unbarred lenticular galaxies is $\sim 0.4$ mag in both blue and infrared light. In other words the absence (or near absence) of luminous barred S0 galaxies is a feature that occurs in both blue light and in infrared classifications. A possible contribution to the small observed difference between the median luminosities of SA0 and SB0 galaxies might be provided by the asymmetry in the effects of small classification errors that results from the fact that there are no barred ellipticals. As a result an E galaxy might be slightly misclassified as an SA0 and an SA0 might be misclassified as an E. However, because there are no barred ellipticals, an SB0 will never be classified as an E. 
It is important to always keep in mind that the classification of early-type galaxies represents a considerable challenge to precision morphology. This is shown most clearly by the rather large scatter between the classification types assigned to the same galaxies by different competent morphologists. Table 4 shows a comparison between the Laurikainen and Sandage \& Tammann classifications of early-type galaxies with Sandage luminosities greater than $M_{B}=-21.5$. The relatively low degree of agreement between these classification types is disappointing. However, for objects fainter than $M_{B}=-21.5$ the agreement appears better with $\sim 2 / 3$ of all galaxies being assigned to the same morphological types by these two sets of authors. It seems that we may have approached the outer boundary of the applicability of morphological classification, where "noise" between individual classifications (and classification systems) becomes a non-negligible factor. Such noise may arise from factors such as differences in display technique (inspection of plates versus computer monitors and NICMOS arrays) or from the presence of subtle inner lenses or shallow core profiles. No two galaxies are identical, so one should not expect all such objects to exhibit the features that define their morphological types with the same strength or in exactly the same way. In this connection it is of interest to note that Sales et al. (2012) have used detailed modeling to show that most spheroidal galaxies consist of superpositions of stellar components with distinct kinematics, ages and metallicities, an arrangement that might survive to the present day because of the paucity of recent major mergers. In particular cold inflows of gas along separate filaments with misaligned spins might settle on off-axis orbits relative to material that had been accreted earlier. The detailed history of gas inflow might therefore affect disk and core formation in ways that lead to small systematic differences between the ways in which these objects are classified by different expert morphologists. 


\section{LUMINOSITIES OF ELLIPTICAL GALAXIES}

Only a small number of galaxies that were classified in the IR by Laurikainen et al. are ellipticals. These objects are typically found to be more luminous that those assigned to type S0. This agrees with previous results by van den Bergh (1998, p.61) and van den Bergh (2011) which clearly show that S0 galaxies are systematically less luminous than either E or Sa galaxies - thus contradicting Hubble's notion that S0 galaxies are truly intermediate between Hubble stages E and Sa. Ellipticals are typically $\sim 2$ times more luminous than lenticulars. As a result E galaxies misclassified as S0s will increase the mean luminosity of the SA0 sample. However, the mean luminosity of SB0 will be unaffected because elliptical galaxies do not have bars and therefore cannot be misclassified as being of type SB0.

\section{DISCUSSION}

The principal results obtained in the present investigation is that luminous unbarred lenticular galaxies are common, whereas such luminous objects are rare or absent among barred lenticulars. This conclusion is found to hold for both galaxies classified in the blue (Sandage \& Tammann 1981) and for those classified in the infrared by Laurikainen et al. (2011). Since elliptical galaxies are, on average, known to be twice as luminous as lenticulars this suggests a possible evolutionary connection between elliptical galaxies and luminous lenticulars. On the other hand the lower luminosity barred lenticular galaxies may, from an evolutionary point of view, be more closely related to spiral galaxies that have been stripped of gas by ram-pressure. A small contribution to the observed $\sim 0.4$ mag. mean luminosity difference between barred and unbarred lenticular galaxies might also be due to the fact that there is an asymmetry in the effects of small morphological classification

errors, which results from the fact that there are no barred elliptical galaxies. As a result an E galaxy might be misclassified as an SA0, but an SB0 would never be misclassified as an 
E. The effects of gas accretion on bar formation (Bournaud \& Combes 2002) are probably not significant for very early-type galaxies, such as those of type SBO.

It is a pleasure to acknowledge helpful exchanges of e-mail with Bob Abraham, Eija Laurikainen, Heikki Salo, Ron Buta and Johan Knapen. I also thank a particularly kind referee. 
Table 1. Luminosity distribution of lenticular galaxies in the catalog of Laurikainen et al.

\begin{tabular}{cccc}
\hline \hline & & & \\
$M_{B}$ & $\mathrm{~N}(\mathrm{SA} 0)$ & $\mathrm{N}(\mathrm{SAB} 0)$ & $\mathrm{N}(\mathrm{SB} 0)$ \\
\hline-22.50 to -22.99 & 1 & 0 & 0 \\
-22.00 to -22.49 & 5 & 1 & 0 \\
-21.50 to -21.99 & 9 & 2 & 0 \\
-21.00 to -21.49 & 6 & 4 & 5 \\
-20.50 to -20.99 & 6 & 6 & 5 \\
-20.00 to -20.49 & 3 & 7 & 7 \\
-19.50 to -19.99 & 7 & 3 & 8 \\
-19.00 to -19.49 & 3 & 1 & 4 \\
-18.50 to -18.99 & 0 & 0 & 2 \\
$>-18.50$ & 1 & 0 & 0 \\
\hline
\end{tabular}

Table 2. Luminosity distribution of SA0, SAB0 and SB0 galaxies (Laurikainen et al.2011) classified in the infrared.

\begin{tabular}{lcll}
\hline \hline & $\mathrm{N}(\mathrm{SA} 0)$ & $\mathrm{N}(\mathrm{SAB} 0)$ & $\mathrm{N}(\mathrm{SB} 0)$ \\
\hline Bright $\left(M_{B}<-21.5\right)$ & 15 & 1 & 0 \\
Faint $\left(M_{B}>-21.5\right)$ & 26 & 23 & 31 \\
\hline
\end{tabular}


Table 3. Luminosity distribution of S0 and SB0 galaxies classified in blue light (Sandage \& Tammann 1981)

\begin{tabular}{ccc}
\hline \hline & $\mathrm{N}(\mathrm{S} 0)$ & $\mathrm{N}(\mathrm{SB} 0)$ \\
\hline Bright $\left(M_{B}<-21.5\right)$ & 16 & 2 \\
Faint $\left(M_{B}>-21.5\right)$ & 96 & 31 \\
\hline
\end{tabular}

Table 4. Comparison between infrared (Laurikainen et al) and blue (Sandage \& Tammannn) classifications of luminous early-type galaxies with $M_{B}<-21.5$

\begin{tabular}{cccc}
\hline \hline S\&T type & E & E/S0 & S0 \\
\hline Laurikainen et al. classification & & & \\
SA0 & 4 & 1 & 7 \\
SAB0 & 0 & 0 & 2 \\
SB0 & 0 & 0 & 0 \\
\hline
\end{tabular}




\section{REFERENCES}

Barway, S., Wadadekar, Y. \& Kembhavi, A. K. 2011, MNRAS, 410, L18

Bournaud, F. \& Combes, F. 2002, A\&A, 392, 83

Buta, R., Corwin, H. G., \& Odewahn, S. C. 2007, The de Vaucouleurs Atlas of Galaxies, Cambridge: Cambridge University Press.

de Vaucouleurs, G. 1959, Handb. der Physik, 53, 275

de Vaucouleurs, G., de Vaucouleurs, A., Corwin, H. G., Buta, R., Paturel, G. \& Fouqué, P. 1991, Third Reference Catalogue of Bright Galaxies, New York: Springer

Djorgovski, S. 1992, in "Morphology and Physical Classification of Galaxies", eds. Longo, G., Capaccioli, M. \& Busarello, G. Dordrecht: Kluwer Acad. p. 427

Hubble, E. 1936, The Realm of the Nebulae, New Haven, Yale University Press, p. 45

King, I. 1992, in "Morphological and Physical Classification of Galaxies" eds. Longo, G., Cappaccioi, M. \& Busarello, Dordrecht, Kluwer Acad. p. 371

Laurikainen, E., Salo, H., Buta, R. \& Knapen, J. H. 2011, MNRAS, 418, 1452

Laurikainen, E., Salo, H., Buta, R. \& Knapen, J. H. 2012, Advances in Astronomy (in press $=\operatorname{arXiv:1111.6447)~}$

Sales, L. V., Navarro, J. F., Theuns, T., Schaye, J., White, S. D. M., Frenk, C. S., Crain, R. A. \& Dalla Vecchia, C. 2012, MNRAS (in press = arXix:1112.2220)

Sandage, A. 1961, The Hubble Atlas of Galaxies, Washington: Carnegie Institution of Washington Publ. No. 618

Sandage, A. \& Bedke, J. 1994, The Carnegie Atlas of Galaxies, Carnegie Institution of Washington Publ. No. 638

Sandage, A. \& Tammann, G. A. 1981, Revised Shapley-Ames Catalog of Bright Galaxies, Washington: Carnegie Institution of Washington 
van den Bergh, S. 1998, Galaxy Morphology and Classification, Cambridg: Cambridge University Press

van den Bergh, S. 2002, AJ, 124, 782 van den Bergh, S. 2011, PASP, 141,188

This manuscript was prepared with the AAS LATEX macros v5.2. 